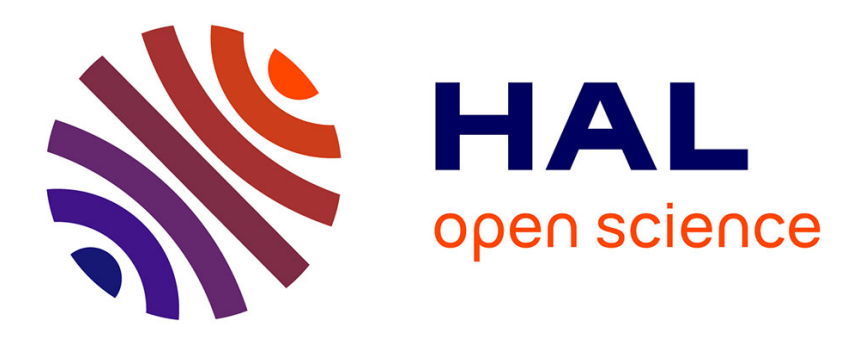

\title{
Direct synthesis of titanium nitride by laser-plasma
}

\author{
A. Thomann, J. Hermann, C. Boulmer-Leborgne
}

\section{To cite this version:}

A. Thomann, J. Hermann, C. Boulmer-Leborgne. Direct synthesis of titanium nitride by laser-plasma. Journal de Physique IV Proceedings, 1994, 04 (C4), pp.C4-55-C4-58. 10.1051/jp4:1994410 . jpa00252548

\section{HAL Id: jpa-00252548 https://hal.science/jpa-00252548}

Submitted on 1 Jan 1994

HAL is a multi-disciplinary open access archive for the deposit and dissemination of scientific research documents, whether they are published or not. The documents may come from teaching and research institutions in France or abroad, or from public or private research centers.
L'archive ouverte pluridisciplinaire HAL, est destinée au dépôt et à la diffusion de documents scientifiques de niveau recherche, publiés ou non, émanant des établissements d'enseignement et de recherche français ou étrangers, des laboratoires publics ou privés. 


\title{
Direct synthesis of titanium nitride by laser-plasma
}

\author{
A.L. THOMANN, J. HERMANN and C. BOULMER-LEBORGNE
}

\author{
GREMI, Université d'Orléans, BP. 6759, 45067 Orléans cedex 2, France
}

\begin{abstract}
Introduction.
Metal nitride thin films are usually deposited on substrates (glass, ceramic, steel) in order to promote their surface characteristics : electrical resistivity, thermal stability, hardness... In many cases, it would be interesting to synthesize the nitride directly on the material surface by chemical reaction with nitrogen. This direct synthesis would avoid the usual problem of film adherence.

In this frame we study the direct nitriding of a metal sample (titanium) submitted to the action of a nitrogen plasma created by a TEA-CO laser beam $(\lambda=10.6 \mu \mathrm{m})$ interacting with the target in a nitrogen atmosphere. Then the synthesized films are analysed by means of different technics. The surface states are controled by scanning electron microscopy (SEM) and rugosity measurements. Chemical composition is investigated on the surface $(50 \AA$ depth) by X-ray photoelectron spectroscopy (XPS), and in the material bulk by Rutherford back-scattering (RBS). Crystalline phases are characterized by X-ray diffraction (XRD).
\end{abstract}

Experimeatal.

A $10.6 \mu \mathrm{m} \mathrm{CO}_{2}$ pulsed laser heam is focused on a $\mathrm{Ti}$ target placed in a chamber containing 760 Torr $\mathrm{N}_{2}$ buffer gas. The target displacements are monitored by a microcomputer. The plasma characterization is achieved by time and space resolved emission spectroscopy.

The temporal shape of the pulses emitted by the laser consists of a prominent first spike followed by a long lasting tail of lower density. It can be varied by changing the $\mathrm{CO}_{2} / \mathrm{N}_{2} \mathrm{ratio}$ in the laser active gas mixture. Thus two kinds of laser pulse shapes have been defined (the short $A$ and the long one B) and are presented in Fig.1. The surface temperature irradiated by the laser for each pulse has been calculated by the 10 heat conduction equation (Fig.2). From this figure it appears that plasma behavior and its coupling with the target depend on the laser pulse shape.

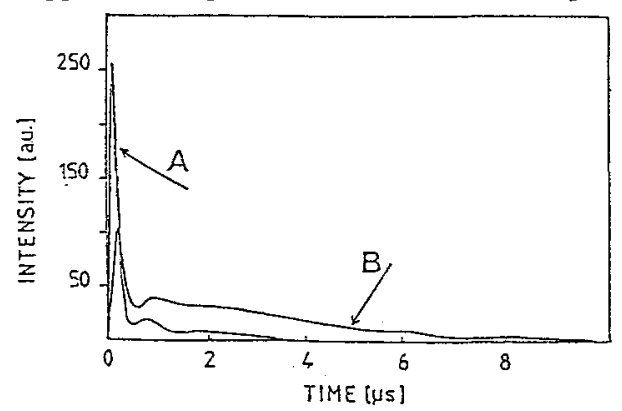

Figure 1. Laser pulse shapes

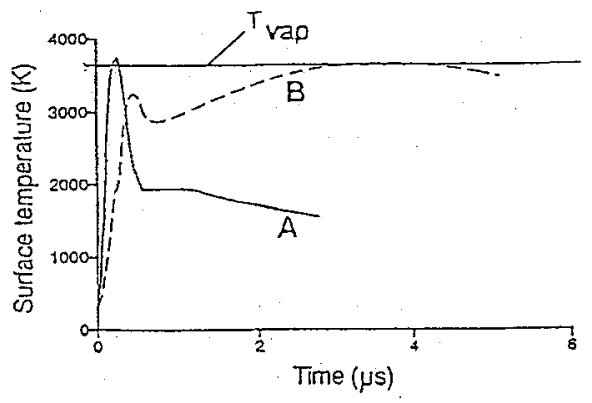

Figure 2. Surface temperatures corresponding to $A$ and $B$ laser pulses

\section{Plasma formation.}

In a first step, the target surface is heated under laser beam action until the vaporization point of surface defects and impurities is reached. Then in a second step, an initial plasma is created in the vapor-gas mixture, close to the irradiated area. Finally this initial plasma induces thermal and shock waves, the plasma expands and is sufficiently dense to absorb the forthcoming laser radiation (by inverse Bremsstrahlung process) and a fully plasma occurs. This plasma duration is $=8 \mu \mathrm{s}$ with a short laser pulse and $\approx 25 \mu \mathrm{s}$ with a long one. Whatever the experimental conditions, no $\mathrm{N}_{2}$ molecular line is observed whereas $\mathrm{N}, \mathrm{N}^{+}$and $\mathrm{N}^{++}$lines are present. It is supposed that $\mathrm{N}_{2}$ is completely dissociated in the first ns of the interaction.

In the case of titanium irradiated by a TEA-CO2 laser beam, it has been checked that the presence of a nitrogen plasma on the metal surface is necessary to obtain a TiN compound. The laser energy threshold to get the breakdown in gas has been determined $\left(20 \mathrm{MW} / \mathrm{cm}^{2)}\right.$. Plasma recoil action yields damage surface, so the experiments were preferentially performed for laser 
energy around this threshold value in order to limit the detonation wave development in the plasma.

Nevertheless the experiment has shown that the time duration of the plasma-target contact is the parameter that controls the TiN synthesis and the damage surface. This stays true in the $20-60 \mathrm{MW} / \mathrm{cm}^{2}$ laser power density range. Indeed, when the target is irradiated by the laser beam, the surface is heated during 100 to 300 laser pulses depending on the laser power density $\left(60-30 \mathrm{MW} / \mathrm{cm}^{2}\right)$; then the initial plasma begins, unsteady lights appear during 200 to 400 pulses ; finally the plasma becomes dense and stable and sticks to the target surface.

In order to understand the mechanisms of TiN synthesis, we have performed experiments for different laser conditions defined above and submitted the targets to different laser irradiation times : from 100 to 1250 laser pulses. Then the surface states have been examined by SEM and roughness measurements, the chemical analysis of the synthesized layer by XPS, RBS and XRD at each step.

Analysis results

\section{Surface states}

SEM photos : Sample submitted to short laser pulse shape, fluence of $28 \mathrm{~J} / \mathrm{cm}^{2}$ and a) 300 pulses b) 450 pulses c) 800 pulses (scale $: 1 \mathrm{~cm} \rightarrow 5 \mu \mathrm{m}$ ).

SEM photos : Sample submitted to short laser pulse shape. fluence of $28 \mathrm{~J} / \mathrm{cm}^{2}$ and a) 300 pulses b) 450 pulses c) 800 pulses (scale : $1 \mathrm{~cm} \rightarrow 5 \mu \mathrm{m}$ ).

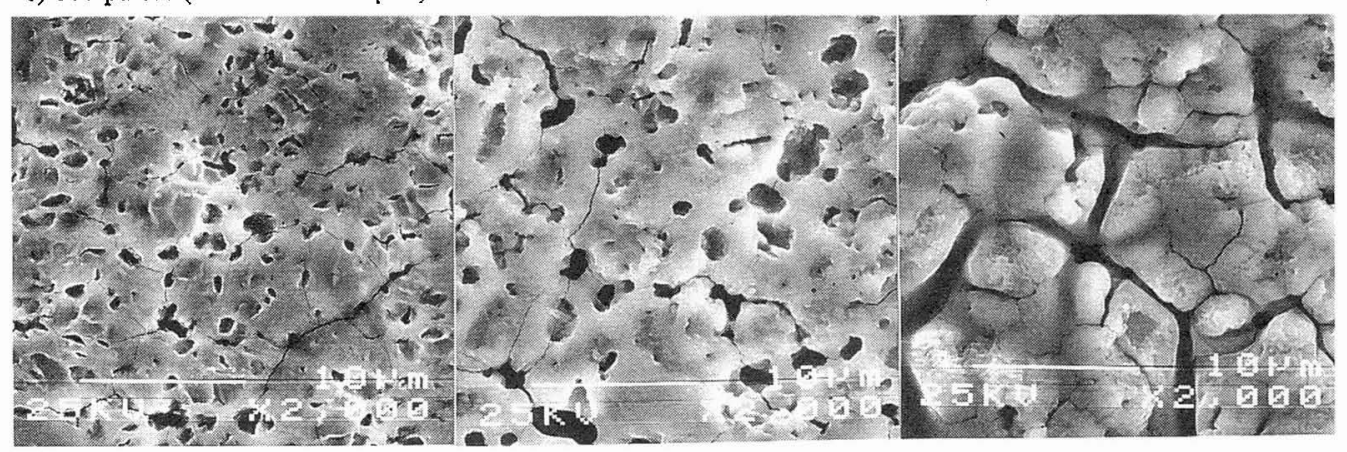

The irradiated sample surface appearence show a variation of colours from grey to gold yellow (typical TiN colour), with the weakest laser pulse number (a), pores and splits appear, for largest ones, the surface is more perturbed (c) with the formation of compact piles and cracks (see SEM photos).

The roughness varies with the laser pulse number from $\pm 0.2 \mu \mathrm{m}$ to $\pm 1.5 \mu \mathrm{m}$ with the short laser pulse shape whatever the laser power density in the $20-60 \mathrm{MW} / \mathrm{cm}^{2}$ range and this roughness is roughiy constant and equals to $\pm 3 \mu \mathrm{m}$ with the long laser pulse shape.

\section{XPS results}

\section{Spectrum}

The program used to fit XPS spectra uses an algorithm based on the convolution of lorentzian and gaussian curves and a Shirley background substraction of experimental signals. The O1s, C1s, N1s and $\mathrm{Ti} 2 \mathrm{p}$ binding energy ranges are examined for the control of the nitriding process and the contamination by oxigen and carbon.

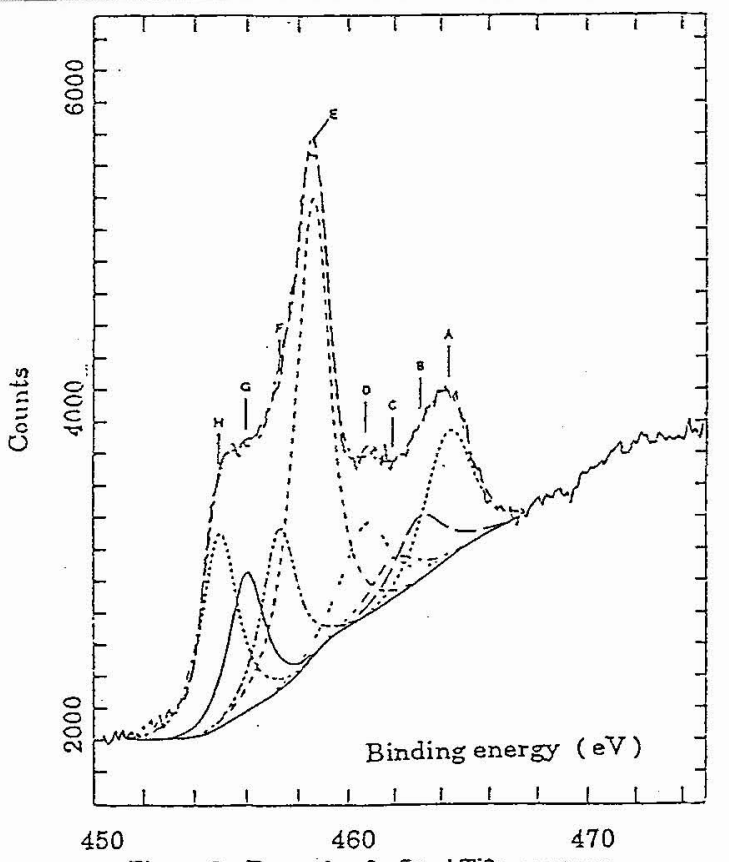

Figure 3: Exemple of a fitted Ti2p spectnm. Sample has received 800 laser pulses with a short pulse shape and a fluence of $23 \mathrm{~J} / \mathrm{cm}^{2}$ 
The XPS analysis and its spectum fit have been achieved for a noirradiated $\mathrm{Ti}$ sample to define a reference spectrum used to resolve the other spectra. In this spectrum region, there are 2 doublets (because of the $\mathrm{Ti} 2 \mathrm{p}_{1 / 2}$ and $\mathrm{Ti} 2 \mathrm{p}_{3 / 2}$ levels) relative to the $\mathrm{Ti}-\mathrm{O}$ bounds in $\mathrm{TiO} 2$ oxide (458.5-464.4eV) and to the metallic bounds $(454.3-460.4 \mathrm{eV})$. This last doublet presents a weaker intensity in comparison with the oxide one, showing that the titanium is covered by an oxide thickness (on o $50 \AA$, the XPS investigation depth).

After laser treatment new species are synthesized, and the Ti2p spectrum is modified. Fig. 3 shows the fitted Ti2p spectrum for a sample submitted to 800 short laser pulses with a fluence of $23 \mathrm{~J} / \mathrm{cm}^{2}$. For calculation, we have supposed that the half-high widths and gaussian/lorentzian coefficients for $\mathrm{Ti} 2 \mathrm{p}_{1 / 2}$ and $\mathrm{Ti} 2 \mathrm{p}_{3 / 2}$ peaks are similar to those of $\mathrm{TiO} 2$. From hanbook data, the energy difference values between the doublet componants are $5.7 \mathrm{eV}$ for $\mathrm{TiO} 2$ and $6.15 \mathrm{eV}$ for $\mathrm{T} i$. Then energy difference betwween the set of doublets is chosen in this value range. Thus three new species are evidenced.
Figure 4: XPS scpectra in the Ti2p region.

Samples have been subnitted to a short laser pulse, with fluence of $23 \mathrm{~J} / \mathrm{cm}^{2}$.

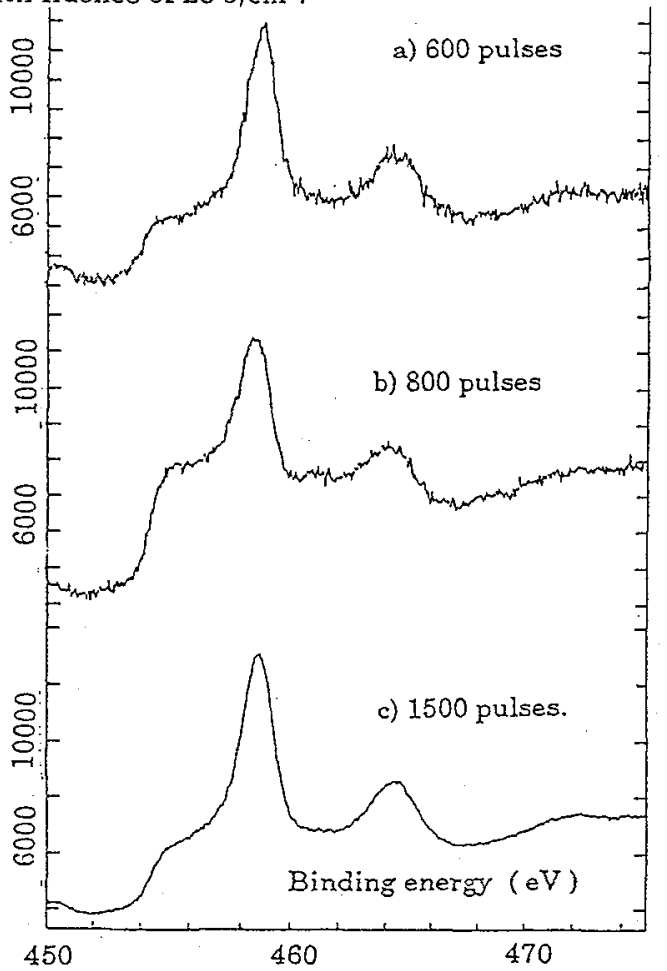

We can see on Fig.4 these new doublets (H-D), (G-C) and (F-B), the peaks (E-A) stand for TiO2 doublet.(H-D) doublet with main peak (Ti2p3/2) located at $455 \mathrm{eV}$ is assumed to be due to titanium nitride (maybe no-stoichiometric). (G-C) and (F-B) peaks are relative to titanium oxinitrides with various oxigen quantities.

Evolution with laser pulse number

Whatever the laser fluence during the target irradiation, the same species are synthesized. That means that the doublets (H-D), (G-C) and (F-B) are always present and located at the same binding energies.

With increasing laser pulse number, the TiN quantity (H-D doublet intensity) seems to decrease in comparison with the oxinitride amount (G-C, F-B doublets). Generally, this TiN doublet intensity is larger for samples submitted to long laser pulse, but the surface roughness of such samples is so high that more attention is given to samples treated with short laser pulses.

Hydrogen amount influence

Native oxide is already present on titanium surface before the treatment as evidenced by XPS spectra, thus hydrogen has been added in the nitrogen ambient gas to reduce oxigen. Various hydrogen percentages have been tested ( 5 to 20\%). XPS analysis show that TiN and oxynitride quantities increase weakly, but no significant difference in their relative amounts appears.

Laser fluence influence

We have performed the experiments for several fluences 16 to $38 \mathrm{~J} / \mathrm{cm}^{2}$ with the short laser pulse shape and the targets have been submitted to different pulse numbers in each case. The same kind of spectrum that the one presented in Fig. 3 is obtained. No significant difference in peak intensities is observed as far as the samples are treated with the same plasma time duration.

Whatever the laser fluence, there is always a pulse number for which TiN and TiNxOy quantity is maximum. When this irradiation time is exceeded, destruction of synthesized TiN and TiOxNy species occurs.

We conclude from this that the most important parameter for an optimum synthesis, is the plasma time duration on the surface. Best results are obtained when the plasma acts several 
seconds on titanium target. Working at high laser fluences allows a faster plasma ignition and a weaker treatment time duration.

\section{RBS results}

The samples obtained with the short laser pulse irradiation at $18 \mathrm{~J} / \mathrm{cm}^{2}$ have been analysed by RBS process with $1.3 \mathrm{Mev}$ $\alpha$ particles. The nitrogen concentration profile in the synthesized layer is deduced from the calculation with rump program fitting the experimental signals. Thus for the lowest laser pulse number (100-200) corresponding to the surface heating, the RBS signal is similar to those of pure Ti sample. At intermediate laser pulse number (300-500), the nitrogen concentration can be represented by a decreasing exponential function of the $\mathrm{z}$ depth from the surface $\mathrm{C}=0.25 \exp \left(-10^{-5} . \mathrm{z}\right)$. That means that there is $3 \mathrm{Ti}$ for $1 \mathrm{~N}$ at the surface $(z=0)$. For larger laser pulse number $(600-800)$, this concentration profile varies to $\mathrm{C}=0.4 \exp \left(-0.7510^{-4} . \mathrm{z}\right)$. The surface TiN stoechiometry becomes $\mathrm{Ti}_{1} \mathrm{~N}_{0.7}$. This holds for larger pulse number. These results show that $\mathrm{N}$ amount in the layer increases with the laser pulse number from 100200 to $600-800$, then saturates for larger number. In addition to XPS results, it can be deduced that the plasma created on the surface yields the nitrogen diffusion into the bulk, this process is limited to a $=3 \mu \mathrm{m}$ thickness layer, for larger plasma time, the surface is damaged and the nitrogen diffusion process stops.

\section{XRD results}

XRD have shown that the layer is polycrystalline; phases of $\alpha-\mathrm{Ti}, \alpha-\mathrm{Ti}$ containing $\mathrm{N}$ and cubic TiN are evidenced .Fig.5 presents the spectrum for a sample obtained after 500 short laser pulse irradiation corresponding to $38 \mathrm{MW} / \mathrm{cm}^{2}$ power density. The spectrum corresponds to a $200 \mathrm{~nm}$ depth investigation. Thus it appears that TiN is synthesized in the bulk and not only on the surface. It must be noted that no oxide or oxinitride are present.

\section{Conclusion}

From these results it appears that the short laser pulse allows a best surface state. As soon as the plasma is ignited, nitrogen reacts with $\mathrm{Ti}$, and $\mathrm{Ti}-\mathrm{N}$ bonds are created in the very close surface $(-100 \AA$ depth) of the target (200-400 laser pulses) whatever the laser power density in the $20-60 \mathrm{MW} / \mathrm{cm}^{2}$ range. Then, if the laser pulse number increases, nitrogen is dissolved in the metal matrix and diffuses in the sample over a thickness of about $3 \mu \mathrm{m}$. This seems to be due to the plasma recoil action. After this step (600-800 laser pulses), no more nitrogen is incorporated but plasma action causes large damages on the surface and the roughness strongly increases.

It has been pointed out that the main parameter to control the TiN synthesis is the time duration plasma interacting with the target. With the highest laser power density (in the 20 $60 \mathrm{~W} / \mathrm{cm} 2$ range) a fast plasma ignition allows a weak time duration for compound synthesis.

The synthesized layer is composed of no-stoichiometric TiN and TiNxOy oxynitrides at the surface. Synthesized layer surface ( $100 \AA$ thickness) exhibits a large oxigen contamination due to $\mathrm{TiO}_{2}$ native oxide on the metal sample, thus we have added various amount of $\mathrm{H}_{2}$ (5 to 20\%) in $\mathrm{N}_{2}$ atmosphere to reduce oxigen. Nevertheless the analysis results do not give clear results allowing a real conclusion and XRD analysis shows that is oxide only a surface contamination.

With short laser pulse, the plasma duration time is sufficient to yield $N$ diffusion in the Ti bulk without creating to much damage surface. Nevertheless, the treatment with long laser pulse gives a large surface roughness as soon as the first hundred pulses act, this is due to the largest plasma time duration obtained with this laser pulse shape.

Vickers microhardness measurements have been achieved on surface samples but the roughness does not yield satisfaisant results, thus we want to try friction resistance tests to valid the utility of this new titanium nitride coating process. 